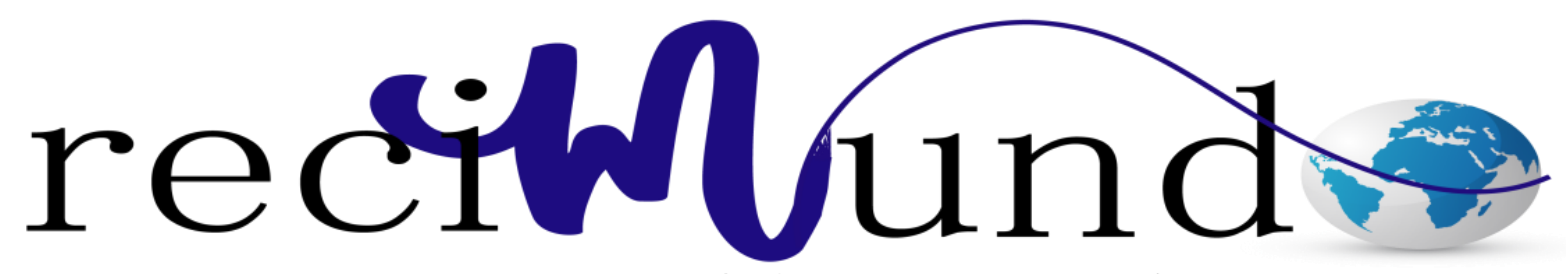

Revista Científica Mundo de la Investigación y el Conocimiento

Carolina Narcisa Chávez Ruiz a ; Luis Fernando Pascual García b; Ximena Melissa Gonzabay Jiménez ${ }^{\mathrm{c}}$; Diana Lilibeth Cruz Yoza ${ }^{\mathrm{d}}$

Parche hemático epidural en el tratamiento de la cefalea postpunción dural

Epidural blood patch in the treatment of post-dural puncture headache

Revista Científica Mundo de la Investigación y el Conocimiento. Vol. 3 núm.3, septiembre, ISSN: 2588-073X, 2019, pp. 31-46

DOI: $10.26820 /$ recimundo/3.(3).septiembre.2019.31-46

URL: http://recimundo.com/index.php/es/article/view/509

Código UNESCO: 3205 Medicina Interna

Tipo de Investigación: Artículo de Revisión

Editorial Saberes del Conocimiento

Recibido: 15/05/2019

Aceptado: 23/06/2019

Publicado: 30/09/2019

Correspondencia: carolina_chavezr@outlook.es

a. Médico; Investigador Independiente; Guayaquil, Ecuador; carolina_chavezr@outlook.es

b. Médico; Investigador Independiente; Guayaquil, Ecuador; luispascualgarcia@ hotmail.es

c. Médico; Investigador Independiente; Guayaquil, Ecuador; goxime311@ hotmail.com

d. Médico; Investigador Independiente; Guayaquil, Ecuador; diana cruz1993@hotmail.com 


\section{Parche hemático epidural en el tratamiento de la cefalea postpunción dural}

Vol. 3, núm. 3., (2019)

Carolina Narcisa Chávez Ruiz; Luis Fernando Pascual García; Ximena Melissa Gonzabay Jiménez; Diana Lilibeth Cruz Yoza

\section{RESUMEN}

Los eventos de cefalalgia experimentados posterior a la realización de una anestesia lumbar epidural para el manejo del dolor durante el trabajo de parto y/o cesárea son correlacionados a la eventual perforación de la duramadre, es una de las complicaciones reportadas con mayor frecuencia, el defecto dural se resuelve de forma espontánea casi siempre. Aún pequeñas cantidades de salida del líquido cefalorraquídeo, pueden provocar cuadros migrañosos fuertes y persistentes, refractarios a cuantos tipos de tratamientos analgésicos se puedan aplicar, hasta que no se remueva la causa primaria, constituida por la descompresión del conducto medular y por ende del sistema ventricular cerebral. Existen diversos protocolos del manejo de cefalea postpunción de duramadre, uno de ellos es de manejo conservador, el cual es una estrategia a corto plazo y está basado en la administración de líquidos, reposo en cama, antinflamatorios no esteroideos, morfina epidural, triptanos y cafeína. El parche hemático epidural (PSE) es un procedimiento para tratar el dolor de cabeza causado por la fuga de fluido cefalorraquídeo. Para crear un parche hemático, el médico inyecta una pequeña cantidad de sangre en el espacio epidural alrededor del canal espinal, cerca del sitio de la punción previa. A medida que la sangre se coagula, forma un "parche" que sella el sitio y detiene la fuga de fluido cefalorraquídeo. En caso de fallo del primer PSE, o si tras un alivio inicial los síntomas reaparecen, se realizará un nuevo PSE, intentando localizar el sitio de la fístula con cisternografía o mielografía para realizar el parche lo más cercano posible a la lesión, otros autores recomiendan aplicar un parche en la región lumbar y si no es efectivo realizar un segundo parche sanguíneo en la zona torácica, demostrando que el parche sanguíneo epidural es un tratamiento eficaz aun cuando no se localice la fuga. La metodología usada es descriptiva, con un enfoque documental, es decir, revisar fuentes disponibles en la red, como google académico, con contenido oportuno y relevante desde el punto de vista científico y actualizado que enriquezca el análisis del tema planteado en este artículo.

Palabras Claves: Derrame de Líquido Cefalorraquídeo; Cefalea Post Punción Dural; Parche Hemático Epidural; Migraña; Tratamiento Conservador no Alivia. 


\title{
Parche hemático epidural en el tratamiento de la cefalea postpunción dural
}

Vol. 3, núm. 3., (2019)

Carolina Narcisa Chávez Ruiz; Luis Fernando Pascual García; Ximena Melissa Gonzabay Jiménez; Diana Lilibeth Cruz Yoza

\begin{abstract}
The events of cephalicgia experienced after performing epidural lumbar anesthesia for pain management during labor and / or caesarean section are correlated to the eventual perforation of the dura, is one of the most frequently reported complications, the defect Dural resolves spontaneously almost always. Even small amounts of cerebrospinal fluid outlet can cause strong and persistent migraine symptoms, refractory to how many types of analgesic treatments can be applied, until the primary cause is removed, consisting of decompression of the medullary canal and hence of the ventricular system cerebral. There are several protocols for the management of post-puncture dural headache, one of which is conservative, which is a short-term strategy and is based on the administration of fluids, bed rest, non-steroidal anti-inflammatory drugs, epidural morphine, triptans and caffeine. The epidural blood patch (PSE) is a procedure to treat the headache caused by the leakage of cerebrospinal fluid. To create a blood patch, the doctor injects a small amount of blood into the epidural space around the spinal canal, near the site of the previous puncture. As the blood coagulates, it forms a "patch" that seals the site and stops the leakage of cerebrospinal fluid. In case of failure of the first PSE, or if after an initial relief the symptoms reappear, a new PSE will be performed, trying to locate the fistula site with cisternography or myelography to make the patch as close as possible to the lesion, other authors recommend apply a patch in the lumbar region and if it is not effective to make a second blood patch in the thoracic area, demonstrating that the epidural blood patch is an effective treatment even if the leak is not located. The methodology used is descriptive, with a documentary approach that is, reviewing sources available on the web, such as google scholar, with timely and relevant content from the scientific and updated point of view that enriches the analysis of the topic raised in this article.
\end{abstract}

Key Words: Spinal Fluid Effusion; Post-Dural Puncture Headache; Epidural Blood Patch; Migraine; Conservative Treatment Does not Relieve. 


\section{Parche hemático epidural en el tratamiento de la cefalea postpunción dural \\ Vol. 3, núm. 3., (2019) \\ Carolina Narcisa Chávez Ruiz; Luis Fernando Pascual García; Ximena Melissa Gonzabay Jiménez; Diana Lilibeth Cruz Yoza}

\section{Introducción.}

Los dolores de cabeza por punción lumbar ocurren frecuentemente en personas que se someten a una punción en la columna vertebral (punción espinal) o a una anestesia intradural. Ambos procedimientos requieren una punción en la membrana dura que recubre la médula espinal y en la raíz de los nervios lumbares y sacros, que se encuentran en la parte inferior de la columna vertebral. La mayoría de los dolores de cabeza por punción se resuelven solos sin ningún tratamiento. Sin embargo, es posible que los dolores de cabeza por punción lumbar intensos que duran más de 24 horas o más necesiten tratamiento.

Los síntomas del dolor de cabeza posterior a una punción lumbar son los siguientes:

- Dolor sordo y pulsátil cuya intensidad varía de leve a incapacitante.

- Dolor que por lo general empeora cuando te sientas o te pones de pie, y que disminuye o desaparece cuando te recuestas.

Acudir al médico si siente dolor de cabeza (migraña) después de someterte a una punción lumbar o de recibir anestesia lumbar, en especial si el dolor de cabeza empeora cuando te sientas o te pones de pie. Los dolores de cabeza por punción lumbar son provocados por la pérdida de líquido cefalorraquídeo a través del orificio de punción en la membrana dura (duramadre) que rodea la médula espinal. Esta pérdida disminuye la presión que ejerce el líquido cefalorraquídeo sobre el cerebro y la médula espinal, lo que produce dolor de cabeza. 


\section{Parche hemático epidural en el tratamiento de la cefalea postpunción dural}

Vol. 3, núm. 3., (2019)

Carolina Narcisa Chávez Ruiz; Luis Fernando Pascual García; Ximena Melissa Gonzabay Jiménez; Diana Lilibeth Cruz Yoza

Existen varios tratamientos para combatir este padecimiento desde los más conservadores como té, analgésicos, reposo, entre otros y otros invasivos como el parche hemático epidural que serán abordados en el presente artículo. En raras ocasiones, el paciente podría correr el riesgo de infección en el área de la inyección. Sin tratamiento, el líquido cefalorraquídeo puede continuar filtrándose. Esto puede aumentar el riesgo de infección. Su dolor de cabeza podría conllevar a un coágulo sanguíneo cerca del cerebro.

\section{Metodología.}

Esta investigación está dirigida al estudio del "Parche hemático epidural en el tratamiento de la cefalea postpunción dural”. Para realizarlo se usó una metodología tipo descriptiva, con un enfoque documental, es decir, revisar fuentes disponibles en la red, como google académico, con contenido oportuno y relevante desde el punto de vista científico para dar respuesta a lo tratado en el presente artículo y que sirvan de inspiración para realizar otros proyectos. Las mismas pueden ser consultadas al final, en la bibliografía.

\section{Resultados.}

La médula espinal está rodeada por tres meninges: duramadre, aracnoides y piamadre. Estas membranas rodean, apoyan y protegen la médula espinal y las raíces nerviosas espinales, incluyendo los de la cola de caballo, y contienen el cristosarcoma filoides (CSF) en la que se suspenden estas estructuras. La duramadre espinal; un tejido conectivo de colágeno vascularizado densa con algunas fibras elásticas, es la membrana más externa que cubre la médula espinal. La duramadre de la médula se separa del hueso periostio cubierto y los 


\section{Parche hemático epidural en el tratamiento de la cefalea postpunción dural}

Vol. 3, núm. 3., (2019)

Carolina Narcisa Chávez Ruiz; Luis Fernando Pascual García; Ximena Melissa Gonzabay Jiménez; Diana Lilibeth Cruz Yoza

ligamentos que forman las paredes del canal vertebral por el espacio epidural (Rodriguez, 2019).

Los eventos de cefalalgia experimentados posterior a la realización de una anestesia lumbar epidural para el manejo del dolor durante el trabajo de parto y/o cesárea son correlacionados a la eventual perforación de la duramadre, con una estadística en la paciente obstétrica ( 0.5 a $2 \%$ de los bloqueos epidurales) del total de las pacientes tratadas con este método de anestesia epidural (Ordaz, Diciembre 2018). Es una de las complicaciones reportadas con mayor frecuencia, el defecto dural se resuelve de forma espontánea casi siempre, suele presentar mayor morbilidad en un gran porcentaje de las pacientes afectadas debido a la cefalea postpunción de duramadre, hasta en el 70 a 85\% de los casos (dependiendo de las características fisiológicas de cada paciente) y la cual en ocasiones resulta ser incapacitante, extendiendo la duración de la estancia hospitalaria.

Los criterios definidos por la International Headache Society para diagnóstico de CPPD se dividen en 4 y utiliza la clínica del paciente (Carrillo, 2016):

- Cefalea intensa, con dolor sordo, no pulsante, generalmente de localización frontooccipital, que empeora los primeros 15 minutos después de levantarse y mejora en 15 minutos después de cambiar a posición decúbito supino; con presencia de al menos uno de los siguientes síntomas: rigidez de cuello, tinnitus, hipoacusia, náuseas y/o fotofobia.

- Que se haya realizado punción lumbar.

- Cefalea dentro de los primeros 5 días posterior a la punción. 


\section{Parche hemático epidural en el tratamiento de la cefalea postpunción dural}

Vol. 3, núm. 3., (2019)

Carolina Narcisa Chávez Ruiz; Luis Fernando Pascual García; Ximena Melissa Gonzabay Jiménez; Diana Lilibeth Cruz Yoza

- Cefalea que remite dentro de la primera semana o 48 horas posteriores a tratamiento eficaz.

Entre las complicaciones comunes se presentan problemas visuales, comúnmente diplopía por disfunción del músculo extraorbicular, parálisis de los nervios intracraneales III, IV y VI, siendo el abducens el más afectado, ya que presenta el trayecto intracraneal más largo. Otra alteración es en la audición como resultado de interferencia entre la interconexión del líquido cefalorraquídeo (LCR) con el espacio perilinfático a través del acueducto coclear.

La fisiopatogénesis de la enfermedad consta en una pérdida de líquido céfalo raquídeo debida al defecto dejado por la accidental perforación de la duramadre con la aguja utilizada para suministrar la sustancia analgésica o anestésica en el espacio epidural, previo a un procedimiento quirúrgico. Aún pequeñas cantidades de salida del líquido cefalorraquídeo, pueden provocar cuadros migrañosos fuertes y persistentes, refractarios a cuantos tipos de tratamientos analgésicos se puedan aplicar, hasta que no se remueva la causa primaria, constituida por la descompresión del conducto medular y por ende del sistema ventricular cerebral.

La sintomatología clásica que se presenta de forma secundaria a punción consiste en cefalea frontooccipital de tipo postural, náusea, vómito, tinnitus, hipoacusia, rigidez de nuca y diplopía, que suele presentarse desde 12 hasta 48 horas después del procedimiento. En algunos estudios se ha encontrado que se presenta con mucha frecuencia cefalea crónica y dolor lumbar posterior asociado a una punción dural con aguja de Tuohy $17 \mathrm{G}$, por lo que se piensa pueden formar parte de la sintomatología (Ubertini, 2013). Los efectos neurológicos adversos debidos a la punción neuroaxial son generalmente transitorios. Complicaciones de tipo infeccioso 


\section{Parche hemático epidural en el tratamiento de la cefalea postpunción dural}

Vol. 3, núm. 3., (2019)

Carolina Narcisa Chávez Ruiz; Luis Fernando Pascual García; Ximena Melissa Gonzabay Jiménez; Diana Lilibeth Cruz Yoza

(arachnoiditis, meningitis y abscesos) son poco probable siempre y cuando se realice una adecuada antisepsia de la piel previa al procedimiento.

Existen diversos protocolos del manejo de cefalea postpunción de duramadre, uno de ellos es de manejo conservador, el cual es una estrategia a corto plazo y está basado en la administración de líquidos, reposo en cama, antinflamatorios no esteroideos, morfina epidural, triptanos y cafeína, medidas que no han mostrado evidencia estadísticamente significativa para disminuir la incidencia de cefalea postpunción, a diferencia del tratamiento basado en el parche hemático, que en la actualidad resulta ser más efectivo y que es considerado como el estándar de oro en el tratamiento.

En los últimos años, se ha reportado el uso de morfina epidural y administración de pregabalina y gabapentina para la reducción de la gravedad del dolor asociado a punción dural con buenos resultados. Existen también reportes del uso de cosintropina, una forma sintética de la hormona adrenocorticotropa, con la cual se observó en un estudio que se llegó a presentar una disminución de hasta el $30 \%$ de las molestias originadas por la punción de duramadre. Sin embargo, ninguno de los tratamientos anteriores ha demostrado tanta efectividad para la remisión de los síntomas como el parche hemático, por tal motivo se podría seguir considerando como el de elección para la remisión definitiva de la sintomatología cuando fracasen las medidas conservadoras o la sintomatología sea muy intensa. La figura $\mathrm{N}^{\circ} 1$ resume el manejo de esta problemática. 


\section{Parche hemático epidural en el tratamiento de la cefalea postpunción dural}

Vol. 3, núm. 3., (2019)

Carolina Narcisa Chávez Ruiz; Luis Fernando Pascual García; Ximena Melissa Gonzabay Jiménez; Diana Lilibeth Cruz Yoza

Figura $\mathbf{N}^{\circ}$ 1. Flujograma propuesto para manejo de pacientes con sospecha de síndrome de hipertensión intracraneal espontánea

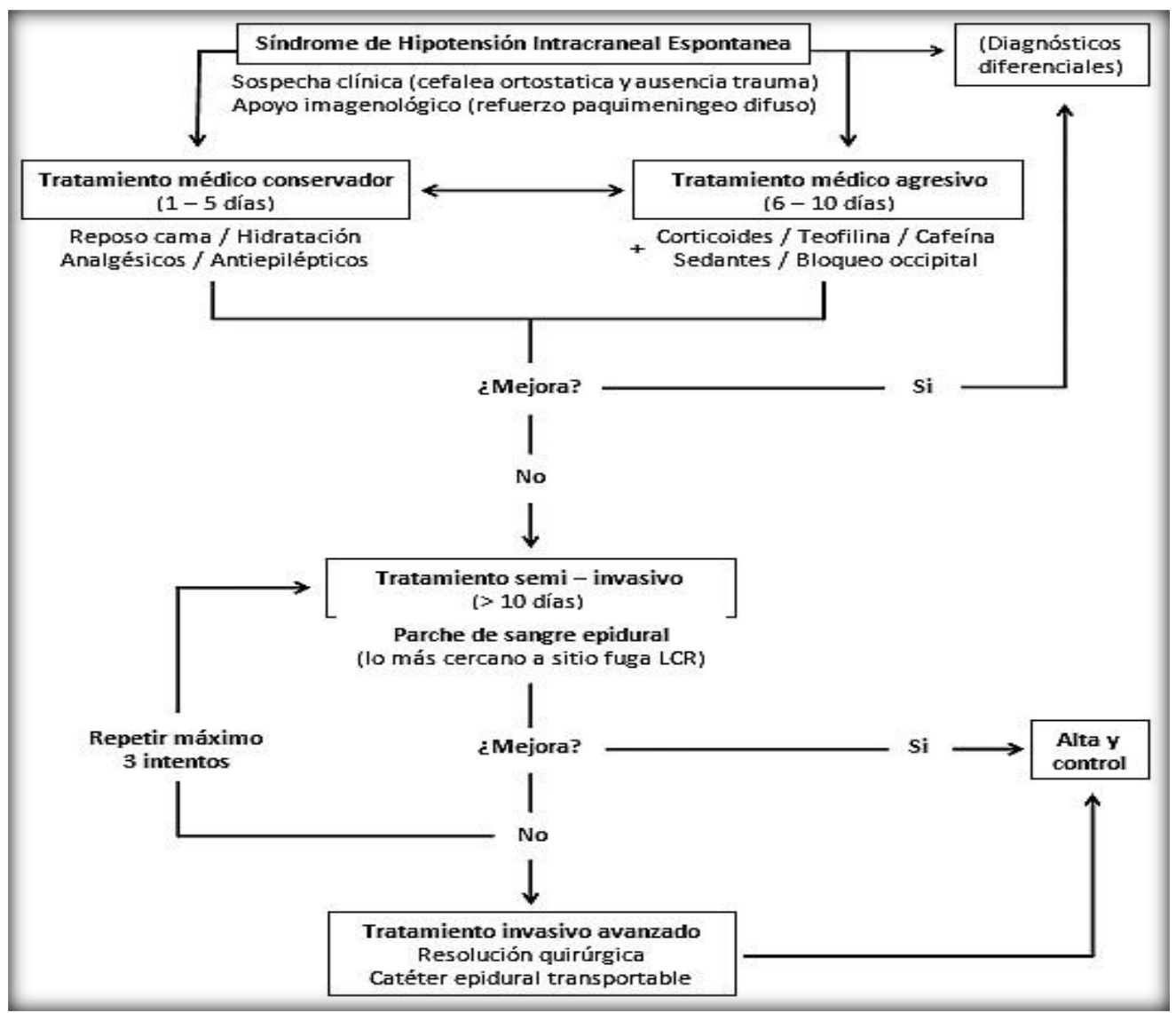

Fuente: (Vidal, 2018)

El efecto terapéutico posterior a la administración del parche hemático suele ser inmediato y se piensa que es condicionado por el aumento transitorio de la presión intracraneal (PIC), la cual se ha establecido tras asociar el aumento del diámetro de la vaina del nervio óptico posterior a la administración de volumen en el espacio epidural, y el efecto a largo plazo tiene que ver con el sellado del defecto dural por la sangre inyectada. (Sesgado, 2011) Existe también la teoría de que el efecto terapéutico único del parche hemático posterior a lesión incidental de 


\section{Parche hemático epidural en el tratamiento de la cefalea postpunción dural}

Vol. 3, núm. 3., (2019)

Carolina Narcisa Chávez Ruiz; Luis Fernando Pascual García; Ximena Melissa Gonzabay Jiménez; Diana Lilibeth Cruz Yoza

duramadre está condicionado únicamente por taponamiento en el defecto dural, el cual se ha precisado mediante resonancia magnética e impide que exista fuga del líquido cefalorraquídeo.

\section{Parche hemático Epidural}

El parche hemático epidural (PSE) es un procedimiento para tratar el dolor de cabeza causado por la fuga de fluido cefalorraquídeo. Esta complicación puede ocurrir después de una punción espinal, una anestesia epidural para el trabajo de parto u otro procedimiento que requiera de punción lumbar. Para crear un parche hemático, el médico inyecta una pequeña cantidad de sangre en el espacio epidural alrededor del canal espinal, cerca del sitio de la punción previa. A medida que la sangre se coagula, forma un "parche" que sella el sitio y detiene la fuga de fluido cefalorraquídeo (Anestesia Chile, 2010).

Figura $\mathbf{N}^{\circ}$ 2. Parche Hemático Epidural

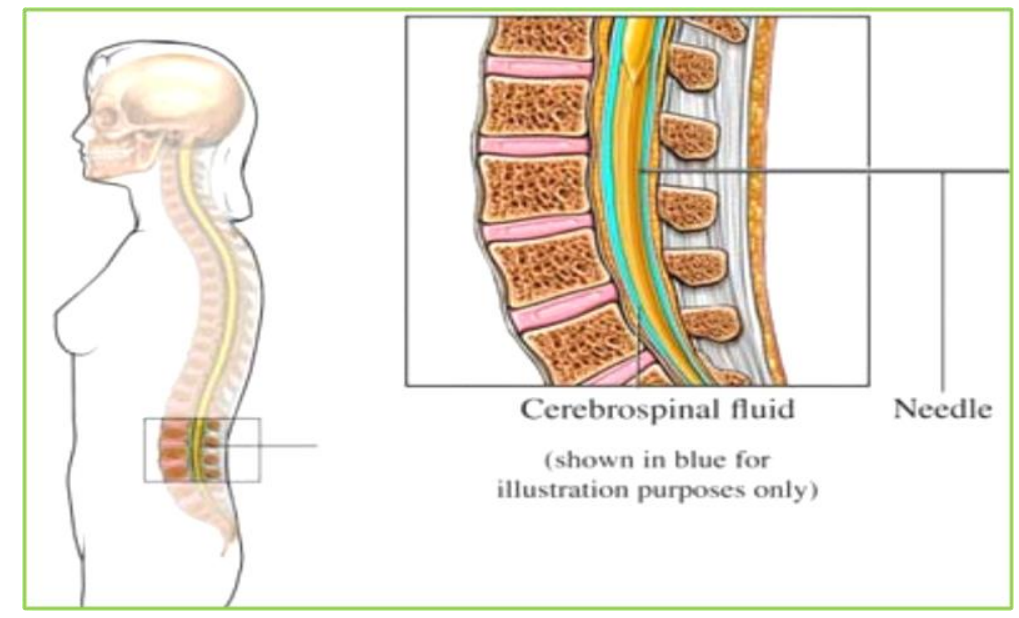

Fuente: (Neff, 2015) 


\section{Parche hemático epidural en el tratamiento de la cefalea postpunción dural}

Vol. 3, núm. 3., (2019)

Carolina Narcisa Chávez Ruiz; Luis Fernando Pascual García; Ximena Melissa Gonzabay Jiménez; Diana Lilibeth Cruz Yoza

Antes del procedimiento

- Su médico le hará una exploración física minuciosa y le explicará los riesgos y beneficios del procedimiento.

- Informe sobre todas las alergias al médico.

- Deberá firmar un consentimiento informado.

- Consiga que alguien lo transporte hasta su casa.

- Se le indicará que no coma ni beba entre 2 y 6 horas antes del procedimiento.

- Si fuera posible, se le indicará que deje de tomar aspirina, antiinflamatorios, ciertos suplementos como por ejemplo vitamina $\mathrm{E}$ y anticoagulantes 10 días antes del procedimiento. Hable sobre la interrupción de estos medicamentos con su médico.

- Posiblemente, se le indicará que evite ciertos suplementos herbarios antes del procedimiento, como el aceite de pescado.

- Tome cualquier otro medicamento de forma habitual.

\section{Descripción del procedimiento}

En la sala de operaciones, se insertarán dos vías intravenosas (agujas) en los brazos para administrar medicamentos y líquidos y para extraer sangre. Posiblemente, le administren un sedante para aliviar la ansiedad. Usted se recostará sobre la camilla boca abajo. El médico le limpiará e higienizará la espalda. Una máquina para radiografías ayudará al médico a dirigir una pequeña aguja al área donde se está perdiendo el líquido cefalorraquídeo. Se inyectará una solución de contraste y se realizarán más radiografías. El médico extraerá una pequeña cantidad de sangre y la inyectará en el área correcta en la columna. Esto coagulará, o sellará, la pérdida. 


\section{Parche hemático epidural en el tratamiento de la cefalea postpunción dural}

Vol. 3, núm. 3., (2019)

Carolina Narcisa Chávez Ruiz; Luis Fernando Pascual García; Ximena Melissa Gonzabay Jiménez; Diana Lilibeth Cruz Yoza

La actividad fibroblástica ocurre dentro de las primeras 48 horas, el depósito de colágeno en 2 semanas y la formación de la cicatriz en tres meses. La cantidad de sangre necesaria para realizar un parche eficaz es variable según los distintos trabajos. Un estudio prospectivo evalúa la eficacia de diferentes volúmenes administrados. Se recomienda entre 10 y $15 \mathrm{ml}$ como volumen suficiente para aumentar la presión de líquido céfalo raquídeo y prevenir su fuga. Este volumen se puede extender de 8 a 10 segmentos del sitio de la colocación (un promedio de seis segmentos en dirección cefálica y tres caudales), con una magnitud media de 1 segmento espinal por cada 1,6 $\mathrm{ml}$ de sangre. Cantidades mayores resultarían innecesarias y podrían estar relacionadas con una mayor incidencia de complicaciones y volúmenes menores de $10 \mathrm{ml}$ con un mayor número de fracasos y recurrencia de la cefalea (López, 2003).

\section{Cuidados después de la cirugía}

En el hospital (Neff, 2015):

- Lo llevarán a una sala de recuperación durante unas horas.

- Le indicarán que permanezca acostado y quieto.

- Lo controlarán y le administrarán líquidos por vía intravenosa.

- Después de unas horas, le indicarán que se levante y se desplace lentamente.

En el hogar

Cuando regrese a casa, haga lo siguiente para ayudar a asegurar una recuperación sin problemas: 


\section{Parche hemático epidural en el tratamiento de la cefalea postpunción dural}

Vol. 3, núm. 3., (2019)

Carolina Narcisa Chávez Ruiz; Luis Fernando Pascual García; Ximena Melissa Gonzabay Jiménez; Diana Lilibeth Cruz Yoza

- Continúe el reposo en la cama y realice movimientos suaves graduales durante las primeras 24 horas.

- Durante los primeros dos o tres días, evite realizar movimientos bruscos, levantar objetos y realizar actividades físicas agotadoras.

- Ingiera muchos líquidos. Puede consumir una dieta normal.

- Retome sus actividades según las tolere.

- Asegúrese de seguir las indicaciones del médico.

Después de salir del hospital, comuníquese con su médico si se presenta cualquiera de las siguientes situaciones:

- Dolor o molestias que no desaparecen (después de 24 horas).

- Signos de infección, incluso fiebre y escalofríos.

- Adormecimiento, debilidad o sensaciones inusuales en las extremidades.

- Problemas para orinar o defecar.

- Cuello rígido.

El parche epidural lumbar es efectivo en el $90 \%$ de los casos, debiendo esperar al menos 24 horas para valorarlo. En caso de fallo del primer PSE, o si tras un alivio inicial los síntomas reaparecen, se realizará un nuevo PSE, intentando localizar el sitio de la fístula con cisternografía o mielografía para realizar el parche lo más cercano posible a la lesión, lo cual ha demostrado tener una mayor efectividad en la resolución de los síntomas. Otros autores recomiendan aplicar un parche en la región lumbar y si no es efectivo realizar un segundo parche sanguíneo en la 


\section{Parche hemático epidural en el tratamiento de la cefalea postpunción dural}

Vol. 3, núm. 3., (2019)

Carolina Narcisa Chávez Ruiz; Luis Fernando Pascual García; Ximena Melissa Gonzabay Jiménez; Diana Lilibeth Cruz Yoza

zona torácica, demostrando que el parche sanguíneo epidural es un tratamiento eficaz aun cuando no se localice la fuga (Carrillo, 2016).

Las tasas de éxito más bajas podrían reflejar punciones dúrales por agujas de mayor calibre. En los estudios obstétricos fue necesario un segundo parche hasta en un $29 \%$ de las pacientes. El PHE debería indicarse en los casos de cefalea moderada o severa resistente al tratamiento conservador más allá de las primeras 24-48 horas. El momento óptimo no se ha determinado aunque la actitud expectante incrementa el tiempo de estancia en el hospital y el riesgo de reingreso. El volumen óptimo de sangre autóloga no se ha determinado. La mayoría de los anestesiólogos reconocen que los 2-3 ml de sangre originalmente descritos por Gormley son insuficientes, y se han propuesto volúmenes medios que oscilan entre 7 y $30 \mathrm{ml}$ (Rodriguez, 2019).

\section{Conclusiones.}

La cefalea postpunción dural es una complicación de la punción de la duramadre realizada para propósitos diagnósticos o terapéuticos o accidentalmente, como una complicación de la anestesia epidural. Es el resultado de un escape de líquido cefalorraquídeo a través del orificio de la duramadre y los consiguientes niveles de líquido reducidos en el cerebro y la médula espinal. El inicio se produce generalmente a los 2 días como cefalea grave e incapacitante y que empeora normalmente con el movimiento, y estando sentado o de pie, y se alivia en cierto modo estando acostado.

La hidratación oral continúa siendo un tratamiento popular para la cefalea postpunción dural y se han probado sustancias como cafeína, cosintropina y sumatriptán. No obstante, el 


\section{Parche hemático epidural en el tratamiento de la cefalea postpunción dural}

Vol. 3, núm. 3., (2019)

Carolina Narcisa Chávez Ruiz; Luis Fernando Pascual García; Ximena Melissa Gonzabay Jiménez; Diana Lilibeth Cruz Yoza

tratamiento conservador es relativamente ineficaz. Por la persistencia y gravedad del malestar conviene aplicar el parche hemático epidural, es un procedimiento para tratar el dolor de cabeza causado por la fuga de fluido cefalorraquídeo. Para crear un parche hemático, el médico inyecta una pequeña cantidad de sangre en el espacio epidural alrededor del canal espinal, cerca del sitio de la punción previa. A medida que la sangre se coagula, forma un "parche" que sella el sitio y detiene la fuga de fluido cefalorraquídeo.

Es importante recordar los factores que propician la cefalea postpunción dural, en especial el calibre de la aguja que se utiliza para la anestesia epidural, puede condicionar sintomatología severa. Las nuevas agujas espinales y epidurales están diseñadas para reducir el tamaño del orificio de la duramadre y tratar de disminuir la fuga de líquido cefalorraquídeo. Las tasas de éxito más bajas podrían reflejar punciones dúrales por agujas de mayor calibre.

En raras ocasiones, el paciente podría correr riesgo de infección en el área de la inyección. Sin tratamiento, el líquido cefalorraquídeo puede continuar filtrándose, esto puede aumentar el riesgo de infección. Su dolor de cabeza podría conllevar a un coágulo sanguíneo cerca del cerebro. Si la fuga es severa, el paciente corre el riesgo de sufrir de convulsiones y hasta podría morir. Podría requerir de otro procedimiento de parche hemático epidural si la cantidad de líquido cefalorraquídeo que se fugó es grande como se explicó en este artículo. Adicionalmente podría requerir de cirugía para reparar a la duramadre. Acuda a su especialista de confianza ante cualquier padecimiento de salud, aclare todas sus dudas referidas a las opciones de tratamiento que mejor se adecuen a su padecimiento. 


\section{Parche hemático epidural en el tratamiento de la cefalea postpunción dural}

Vol. 3, núm. 3., (2019)

Carolina Narcisa Chávez Ruiz; Luis Fernando Pascual García; Ximena Melissa Gonzabay Jiménez; Diana Lilibeth Cruz Yoza

\section{Bibliografía.}

Anestesia Chile. (19 de Abril de 2010). ¿Qué es un parche de sangre? Obtenido de Anestesia Chile: https://www.anestesiachile.cl

Carrillo, O. (2016). Protocolo de tratamiento para la cefalea postpunción de duramadre. Revista Mexicana de Anestesiología, 39(3), 205-212.

López, F. (2003). Parche sanguíneo epidural como tratamiento de un cuadro de hipotensión intracraneal espontánea. Revista de la Sociedad Española del Dolor, 10(8), 511 - 515.

Neff, D. (Junio de 2015). Parche Sanguíneo Epidural. Obtenido de Cancer Care of Western New York: https://www.cancercarewny.com

Ordaz, A. (Diciembre 2018). Volúmenes bajos para la realización de parche hemático como tratamiento defi nitivo de la cefalea postpunción de duramadre en pacientes obstétricas mexicanas. Revista Mexicana de Anestesiología Vol. 41. No. 4, 263 - 267 https://www.medigraphic.com.

Rodriguez, G. (2019). Tratamiento en pacientes con cefalea postpunción dural. Revista Científica de Investigación actualización del mundo de las Ciencias, 3(3), 821 - 841.

Sesgado, M. (2011). Analgesia epidural obstétrica: fallos y complicaciones neurológicas de la técnica. Revista de la Sociedad Española del Dolor,Volumen.18 No.5, $\mathrm{http} / / / \mathrm{scielo}$.isciii.es.

Ubertini, A. (2013). Parche hematico epidural en migraña post anestesia epidural y neuro axial: riesgos versus beneficios. Revista medica de costa rica y centroamerica, LXX(605), 5 - 7.

Vidal, P. (2018). Parche de sangre epidural torácico, de alto volumen, para el tratamiento del Síndrome de Hipotensión Intracraneana Espontánea secundario a fuga cervical de líquido cefalorraquídeo, ¿mejora el período asintomático?. Reporte de caso. Revista Chilena Anestesiologia, 47(1), 31 - 36. 